\title{
Error Performances of Multiple Access System Using Analog Network Coding
}

\author{
Wei Guan, K. J. Ray Liu \\ Department of Electrical and Computer Engineering \\ University of Maryland, College Park, MD 20742 \\ Email: \{wguan, kjrliu\}@umd.edu
}

\begin{abstract}
In this work, we study the error performances of the multiple access system using analog network coding (ANC), where all the users broadcast their data to the relays and destination simultaneously in the first phase, and the relays forward the source messages that have been mixed in the air in the second phase. Depending on the type of relay power constraint, both the variable gain relaying and fixed gain relaying are considered. We show that the error rates of both schemes scale as $O\left(\frac{\log P}{P^{2}}\right)$ at high signal-to-noise ratio (SNR), where $P$ is the transmitted power. We also show that the error performance is dominated by the relay-destination channel, thus the best relay position should be close to the destination. We also demonstrate that ANC may be outperformed by the conventional amplify-andforward scheme when the data rate is low and the user number is not large. Finally, we propose a relay selection strategy based on Min-Max criterion that can achieve full diversity when there are multiple relays.
\end{abstract}

\section{INTRODUCTION}

Cooperative communications have gained much attention recently as a way to provide spatial diversity, extend transmission coverage and save power consumption [1]. The two most popular cooperation protocols are amplify-and-forward (AF) and decode-and-forward (DF), both of which are based on repetition coding [2]. The error performances of these two protocols have been widely discussed in [3][4], which demonstrate the significant gain of node cooperation over the traditional point-to-point communication system.

In practice, many user devices are subject to half-duplex constraints. So the above two protocols have the shortcomings of low spectral efficiency, as two orthogonal channels are needed to complete the whole message delivery. Wireless network coding has thus been developed to address such issues [5][6]. Different from conventional relays which simply reproduce and forward the data of each source individually, the network-coded relays first mix different source messages and then forward a single packet that provides side information for all sources. Based on $\mathrm{AF}$ and $\mathrm{DF}$, there are generally two kinds of wireless network coding schemes, i.e., analog network coding (ANC) [5] and physical-layer network coding (PLNC) [6]. In PLNC, the coding is performed in the finite field; however, only half of the degrees of spatial diversity may be leveraged when there exists random decoding error at the relays [7], and special relaying and detection schemes have to be employed to achieve full diversity [8], which complicates the associated transceiver design. On the other hand, in ANC the source messages are directly combined in the signal space and consequently no relay decoding is required.

ANC has been widely used in the multiple-access relay channel due to its simplicity. For example, [9] studied the channel capacity and developed a relay selection scheme; [10] derived the corresponding diversity-multiplexing tradeoff (DMT) curve; [11] devised the space-time coding schemes to achieve the optimal DMT; and [12] obtained the diversity bound of the distributed beamforming strategy with quantized feedback.

We remark that most of the above literatures [9]-[11] are from an information-theoretic view, which assumes Gaussian input and the codeword is infinitely long. However, for systems using practical modulations, the designers usually care more about the corresponding error performances so as to evaluate the practical network performances. Although [12] derived the diversity performances of error rate, it focuses only on the instantaneous relay power constraint, and the coding gain is still unclear. So in this work, we study the error performances of a general multiple access system assisted by ANC relays, subject to either instantaneous power constraint or average power constraint. We first derive the pairwise error probability (PEP) of a single-relay system, and show that a diversity order of 2 can be achieved. We demonstrate that the PEP is dominated by the relay-destination channel, thus the best relay position should be close to the destination. We also reveal that the conventional AF system may outperform ANC when the data rate is low. Finally, we propose a relay selection strategy based on Min-Max criterion, and prove that it can achieve full diversity when there are multiple relays.

Notations: $|\cdot|$ and $(\cdot)^{*}$ stands for absolute value and conjugate, respectively. We shall use abbreviation i.i.d. for independent and identically distributed, and denote $Z \sim \mathcal{C N}\left(\mu, \sigma^{2}\right)$ as a circularly symmetric complex Gaussian random variable with the real part and imaginary part being i.i.d. and satisfying $\mathcal{N}\left(\mu, \frac{\sigma^{2}}{2}\right)$. The probability of an event $\mathcal{A}$ and the cumulative distribution function (CDF) of a random variable $Z$ is denoted by $\operatorname{Pr}(\mathcal{A})$ and $F(z)$, respectively. We define $Q(x)=\frac{1}{\sqrt{2 \pi}} \int_{x}^{\infty} e^{-\frac{t^{2}}{2}} d t$ as the Q-function. Finally, we say $h(x)=\mathrm{O}(g(x))$ if $\limsup _{x \rightarrow \infty} \frac{h(x)}{g(x)}<\infty$.

\section{SYSTEM MODEL WITH SINGLE-RELAY}

Consider a symmetric multiple access channel with $K(\geq 2)$ source nodes sending data to a common destination with the 


$$
\begin{aligned}
F_{W_{1}}(w) \leq & 1-\exp \left(-\frac{a+b}{a b} w\right) 2 \sqrt{\frac{w+w^{2}}{a b}} K_{1}\left(2 \sqrt{\frac{w+w^{2}}{a b}}\right) \\
& +\frac{N c w}{a b} \exp \left(-\frac{a+b-c}{a b} w\right) E_{1}\left(\frac{c w}{a b}\right) \stackrel{w \ll 1}{\approx} \frac{N c+1}{a b} w \log \frac{1}{w}, \\
F_{W_{2}}(w)= & 1-\exp \left(-\frac{w}{b}\right) \sqrt{\frac{4 w c}{a b}} K_{1}\left(\sqrt{\frac{4 w c}{a b}}\right) \stackrel{w \ll 1}{\approx} \frac{c}{a b} w \log \frac{1}{w} .
\end{aligned}
$$

help of a single relay node. In the first phase, all the source nodes broadcast their data simultaneously. Suppose perfect synchronization among the transmitters, the received signal at the relay and at the destination can be represented as

$$
\left\{\begin{array}{l}
y_{s r}=\sqrt{P \lambda_{s r}} \sum_{k=1}^{K} f_{k} s_{k}+n_{s r}, \\
y_{s d}=\sqrt{P \lambda_{s d}} \sum_{k=1}^{K} h_{k} s_{k}+n_{s d}
\end{array}\right.
$$

respectively. Here $n_{s r}, n_{s d} \sim \mathcal{C N}(0,1)$ are the additive noise; $f_{k}, h_{k} \sim C N(0,1)$ are the rayleigh fading channel with the channel gain being $\lambda_{s r}$ and $\lambda_{s d}$, respectively; $P$ is the transmitted power; and $s_{k}$ is the source symbol chosen from some constellation $\Omega$ with normalized power, i.e., $E\left|s_{k}\right|^{2}=1$. Then in the second phase, the relay node shall forward the received messages to the destination with the transmitted signal being $x_{r}=\sqrt{\alpha P} y_{s r}$, where $\alpha$ is the amplification factor. If instantaneous relay power constraint is imposed, the amplification factor is

$$
\alpha_{V G R}=\frac{1}{P \lambda_{s r} \sum_{k=1}^{K}\left|f_{k}\right|^{2}+1},
$$

which is called variable gain relaying (VGR) as $\alpha_{V G R}$ is adaptive to the real-time input channel conditions. On the other hand, if the average relay power is limited by $P$ in the long run, then

$$
\alpha_{F G R}=\frac{1}{K P \lambda_{s r}+1},
$$

which is called fixed gain relaying (FGR). In both cases, the received signal at the destination is

$$
y_{r d}=\sqrt{\lambda_{r d}} g x_{r}+n_{r d}=\sqrt{\alpha P^{2} \lambda_{s r} \lambda_{r d}} g \sum_{k=1}^{K} f_{k} s_{k}+\tilde{n}_{r d},
$$

where $\tilde{n}_{r d} \triangleq \sqrt{\alpha P \lambda_{r d}} g n_{s r}+n_{r d} \sim \mathcal{C N}\left(0, \alpha P \lambda_{r d}|g|^{2}+1\right)$ is the equivalent additive noise, and $g \sim C N(0,1)$ is the rayleigh fading channel with the channel gain being $\lambda_{r d}$. Upon observing the signal $y_{s d}$ and $y_{r d}$, the destination can perform maximum-likelihood detection to jointly decode all the source symbols as

$$
\begin{aligned}
\mathbf{s}_{d}=\arg \min _{\hat{s}_{k} \in \Omega}\left|y_{s d}-\sqrt{P \lambda_{s d}} \sum_{k=1}^{K} h_{k} \hat{s}_{k}\right|^{2} \\
+\frac{\left|y_{r d}-\sqrt{\alpha P^{2} \lambda_{s r} \lambda_{r d}} g \sum_{k=1}^{K} f_{k} \hat{s}_{k}\right|^{2}}{\alpha P \lambda_{r d}|g|^{2}+1} .
\end{aligned}
$$

\section{ERROR PERFORMANCES ANALYSIS}

In this section, we analyze the error performances of the above-mentioned protocol. After some manipulations, we can bound the conditional PEP as

$$
\operatorname{Pr}(\mathbf{s} \rightarrow \hat{\mathbf{s}}) \leq E\left[\exp \left(-\left(W_{d}+W_{r}\right)\right)\right],
$$

where $W_{d}=\frac{1}{4} P \lambda_{s d}\left|\mathbf{h}^{T} \Delta \mathbf{s}\right|^{2}, W_{r}=\frac{\alpha P^{2} \lambda_{s r} \lambda_{r d}|g|^{2}\left|\mathbf{f}^{T} \Delta \mathbf{s}\right|^{2}}{4\left(\alpha P \lambda_{r d}|g|^{2}+1\right)}$, $\mathbf{h}=\left(h_{1}, h_{2}, \cdots, h_{K}\right)^{T}, \mathbf{f}=\left(f_{1}, f_{2}, \cdots, f_{K}\right)^{T}, \Delta \mathbf{s}=\mathbf{s}-\hat{\mathbf{s}}$, and we have applied Chernoff bound [1] in the inequality. As $W_{d}$ follows exponential distribution, we have

$$
E\left[\exp \left(-W_{d}\right)\right]=\frac{4}{P \lambda_{s d}\|\Delta \mathbf{s}\|^{2}+4} \stackrel{P \rightarrow \infty}{\approx} \frac{4}{\lambda_{s d}\|\Delta \mathbf{s}\|^{2}} P^{-1} .
$$

To evaluate the expectation of the second term in (6), we use the following lemma.

Lemma 1: Let $W_{1}=\frac{a b X Y}{a X+b Y+c Z+1}$ and $W_{2}=\frac{a b X Y}{a X+c}$, where $a, b$ and $c$ are constants, $X$ and $Y$ are independent exponential random variables with unit mean, and $Z$ is independent of $X$ and $Y$ and has the gamma distribution $f(z)=\frac{1}{\Gamma(N)} z^{N-1} e^{-z}$, then for $w \geq 0$ the CDF of $W_{1}$ and $W_{2}$ is given respectively by (8) and (9) shown on the top of this page, where $E_{1}(z)$ is the exponential integral function [13, 5.1.1] and $K_{1}(z)$ is the first-order modified Bessel function of the second kind [13, 9.6.1].

Proof: See [15].

For FGR, after plugging (3) in $W_{r}$, we have $W_{r, F G R}=$ $\frac{P^{2} \lambda_{s r} \lambda_{r d}|g|^{2}\left|\mathbf{f}^{T} \Delta \mathbf{s}\right|^{2}}{4\left(P \lambda_{r d}|g|^{2}+K P \lambda_{s r}+1\right)}$. According to Lemma 1, the CDF of $W_{r, F G R}$ can be obtained by substituting $a_{1}=P \lambda_{r d}, b_{1}=$ $\frac{1}{4} P \lambda_{s r}\|\Delta \mathbf{s}\|^{2}$, and $c_{1}=K P \lambda_{s r}+1$ in (9). Then we can obtain (10) shown on the top of next page, where we use $[14,6.643 .3],[13,13.1 .33]$ and $[13,13.2 .5]$ in $(a)$, and the inequality $E_{1}(z)<e^{-z} \log \left(1+\frac{1}{z}\right)[13,5.1 .20]$ in $(b)$. 


$$
\begin{aligned}
E\left[\exp \left(-W_{r, F G R}\right)\right] & \stackrel{(a)}{=} \frac{1}{b_{1}+1}+\frac{b_{1} c_{1}}{a_{1}\left(b_{1}+1\right)^{2}} \exp \left(\frac{c_{1}}{a_{1}\left(b_{1}+1\right)}\right) E_{1}\left(\frac{c_{1}}{a_{1}\left(b_{1}+1\right)}\right) \\
& \stackrel{(b)}{\leq} \frac{1}{b_{1}+1}+\frac{b_{1} c_{1}}{a_{1}\left(b_{1}+1\right)^{2}} \log \left(1+\frac{a_{1}\left(b_{1}+1\right)}{c_{1}}\right) \stackrel{P \rightarrow \infty}{{ }^{\infty}} \frac{4 K}{\lambda_{r d}\|\Delta \mathbf{s}\|^{2}} \frac{\log P}{P}
\end{aligned}
$$

$$
\begin{aligned}
& E\left[\exp \left(-W_{r, V G R}\right)\right] \stackrel{(a)}{\leq} 1-\frac{\|\Delta \mathbf{s}\|^{2}}{4} \exp \left(-\frac{1}{\sqrt{a_{2} b_{2}}}\right)\left(\frac{\|\Delta \mathbf{s}\|^{2}}{4}+\frac{\left(\sqrt{a_{2}}+\sqrt{b_{2}}\right)^{2}}{a_{2} b_{2}}\right)^{-1} \\
& \quad+\frac{(K-1) a_{2}\|\Delta \mathbf{s}\|^{2}}{4}\left(\frac{16}{\|\Delta \mathbf{s}\|^{4} a_{2}^{2}} \log \left(1+\frac{\|\Delta \mathbf{s}\|^{2} a_{2}}{4}\right)-\frac{1}{\frac{\|\Delta \mathbf{s}\|^{2} a_{2}}{4}\left(1+\frac{\|\Delta \mathbf{s}\|^{2} a_{2}}{4}\right)}\right) \stackrel{P \rightarrow \infty}{\approx} \frac{4(K-1)}{\lambda_{r d}\|\Delta \mathbf{s}\|^{2}} \frac{\log P}{P},
\end{aligned}
$$

Combining (7) with (10) leads to

$$
\operatorname{Pr}(\mathbf{s} \rightarrow \hat{\mathbf{s}} \mid \mathrm{FGR}) \leq \frac{16 K}{\lambda_{r d} \lambda_{s d} d_{\min }^{4}} \frac{\log P}{P^{2}}
$$

as $P \rightarrow \infty$, where $d_{\min }=\min _{s, \hat{s} \in \Omega, s \neq \hat{s}}|s-\hat{s}|$ is the minimum distance of any two distinct points in the set $\Omega$.

Next we study the PEP of VGR. After plugging (2) in $W_{r}$, we have $W_{r, V G R}=\frac{P^{2} \lambda_{s r} \lambda_{r d}|g|^{2}\left|\mathbf{f}^{T} \Delta \mathbf{s}\right|^{2}}{4\left(P \lambda_{r d}|g|^{2}+P \lambda_{s r} \sum_{k=1}^{K}\left|f_{k}\right|^{2}+1\right)}$. For any error vector $\Delta \mathbf{s} \neq 0$, we can always find a $K \times K$ unitary matrix $\mathbf{U}$ with the first row being $\frac{\Delta \mathbf{s}^{T}}{\|\Delta \mathbf{s}\|}$. Define a new vector $\tilde{\mathbf{f}}=\mathbf{U f}$, then we have $W_{r, V G R}=\frac{\|\Delta \mathrm{s}\|^{2}}{4} \tilde{W}_{r, V G R}$ with $\tilde{W}_{r, V G R}=$ $\frac{P^{2} \lambda_{s r} \lambda_{r d}|g|^{2}\left|\tilde{f}_{1}\right|^{2}}{P \lambda_{r d}|g|^{2}+P \lambda_{s r}\left|\tilde{f}_{1}\right|^{2}+P \lambda_{s r} \sum_{k=2}^{K}\left|\tilde{f}_{k}\right|^{2}+1}$. As $\tilde{\mathbf{f}} \sim \mathcal{C N}(\mathbf{0}, \mathbf{I}),\left|\tilde{f}_{1}\right|^{2}$ is independent of $\sum_{k=2}^{K}\left|\tilde{f}_{k}\right|^{2}$, which has the gamma distribution. According to Lemma 1, the CDF of $\tilde{W}_{r, V G R}$ can be obtained after plugging $a_{2}=P \lambda_{r d}, b_{2}=c_{2}=P \lambda_{s r}$ and $N=K-1$ into (8). Then we can obtain (12) shown on the top of this page, where we use $[14,6.227 .1]$ and the inequality $x K_{1}(x) \geq \exp (-x)$ [12] in (a). Combining (7) with (12) leads to

$$
\operatorname{Pr}(\mathbf{s} \rightarrow \hat{\mathbf{s}} \mid \text { VGR }) \leq \frac{16(K-1)}{\lambda_{r d} \lambda_{s d} d_{\min }^{4}} \frac{\log P}{P^{2}}
$$

as $P \rightarrow \infty$.

Remark 1: It is observed that the PEPs scale as $\mathrm{O}\left(\frac{\log P}{P^{2}}\right)$ at high SNRs, thus both of FGR and VGR can achieve a diversity order of 2. However, the multi-user interferences (MUI) at the intended receiver introduce an additional logarithmic term (i.e., $\log P$ ) in the expression of PEP, which would incur noticeable diversity loss in the reasonably high SNR regions. Recall that the multiple access could also be accomplished by the conventional repetition-coding based AF protocol, which serves a single user each time and as a result a total of $4 K$ orthogonal channels is required. The error rate of AF protocol scales as $\mathrm{O}\left(P^{-2}\right)$ for VGR [3] and $\mathrm{O}\left(\frac{\log P}{P^{2}}\right)$ for FGR [4], respectively. Therefore, ANC is generally more bandwidth efficient than the conventional AF; however, as the error rate of AF-VGR decreases faster, ANC may even be outperformed by AF-VGR when the data rate is low and the user number $K$ is not large.

Remark 2: By comparing (11) and (13), we observe that increasing the user number $K$ would not degrade the diversity gain. However, since the PEP is proportional to $K$, there is a linear loss of coding gain. Relatively speaking, VGR performs slightly better than FGR. This is because the instantaneous output power at the relay node is always normalized to $P$ in VGR, which helps to mitigate the extent of channel fading; however, such performance gap would diminish as $K \rightarrow \infty$. It is also noteworthy that the PEP is inversely proportional to $\lambda_{r d}$ but is independent of $\lambda_{s r}$. Consequently, the quality of relaydestination channel dominates the overall error performances, and the best relay position should be close to the destination.

\section{RELAY SELECTION STRATEGY}

Yet we focus only on the system with a single relay. In a large wireless network with multiple relays, it is possible to further improve diversity performances by leveraging spatial diversity through relay selection. So in this section, we study the relay selection strategy that can achieve full diversity.

The system model is a natural extension of the single-relay model discussed in the last section. Suppose there are now $L$ parallel relays. Let $f_{k l}$ be the channel coefficient from the $k$ th user to the $l$ th relay, and $g_{l}$ be the channel from the $l$ th relay to the destination. We still consider a symmetric network, where all the user-relay channels have the same pathloss coefficient $\lambda_{s r}$, and all the relay-destination channels have the same path-loss coefficient $\lambda_{r d}$. Suppose only one good relay (i.e., the $q$ th relay) is selected to help forward the source messages. The resulting signal model is basically the same as that in the single-relay case after properly modifying the subscripts in (1a) and (4). To be specific, all the users still broadcast concurrently in the first phase. The received signal at the $q$ th relay is $y_{s r_{q}}=\sqrt{P \lambda_{s r}} \sum_{k=1}^{K} f_{k q} s_{k}+n_{s r_{q}}$, and the received signal at the destination is still given by (1b). Then in the second phase, the $q$ th relay will amplify and forward the data using either $\alpha_{q, V G R}=\left(P \lambda_{s r} \sum_{k=1}^{K}\left|f_{k q}\right|^{2}+1\right)^{-1}$ or 
$\alpha_{q, F G R}=\left(K P G \lambda_{s r}+1\right)^{-1}$. Note that $\alpha_{q, F G R}$ is slightly different from (3), as the input channel distribution has changed after relay selection. The constant $G(>1)$ can be obtained using computer simulations once the relay selection scheme is given, and this factor would not influence the diversity performance. The received signal at the destination during the second phase is $y_{r_{q} d}=\sqrt{\alpha P^{2} \lambda_{s r} \lambda_{r d}} g_{q} \sum_{k=1}^{K} f_{k q} s_{k}+\tilde{n}_{r_{q} d}$, where $\tilde{n}_{r_{q} d} \sim \mathcal{C N}\left(0, \alpha P \lambda_{r d}\left|g_{q}\right|^{2}+1\right)$. Finally, the MLD similar to (5) is performed to decode all the user symbols based on the observations $y_{s d}$ and $y_{r_{q} d}$, and the conditional PEP is given by

$$
\operatorname{Pr}\left(\mathbf{s} \rightarrow \hat{\mathbf{s}} \mid \mathbf{h}, \mathbf{f}_{s q}, g_{q}\right) \leq \exp \left(-W_{d}-W_{r_{q}}(\Delta \mathbf{s})\right),
$$

where $W_{r_{q}}(\Delta \mathbf{s})=\frac{\alpha P^{2} \lambda_{s r} \lambda_{r d}\left|g_{q}\right|^{2}\left|\mathbf{f}_{s q}^{T} \Delta \mathbf{s}\right|^{2}}{4\left(\alpha P \lambda_{r d}\left|g_{q}\right|^{2}+1\right)}, \quad W_{d}=$ $\frac{1}{4} P \lambda_{s d}\left|\mathbf{h}^{T} \Delta \mathbf{s}\right|^{2}$ and $\mathbf{f}_{s q}=\left(f_{1 q}, f_{2 q}, \cdots, f_{K q}\right)^{T}$. It is observed that the quality of the $q$ th relay branch is uniquely characterized by $W_{r_{q}}(\Delta \mathbf{s})$, which itself depends on the error vector $\Delta \mathbf{s}$. As the real error probability is lower bounded by any PEP, we propose to select the relay branch that can minimize the maximum PEP. Since the exponential function is monotonically decreasing, the above Min-Max relay selection strategy can be equivalently formulated as

$$
\begin{aligned}
q & =\arg \min _{l=1,2, \cdots, L} \max _{\hat{\mathbf{s}} \neq \mathbf{s}} \operatorname{Pr}\left(\mathbf{s} \rightarrow \hat{\mathbf{s}} \mid \mathbf{h}, \mathbf{f}_{s l}, g_{l}\right) \\
& =\arg \max _{l=1,2, \cdots, L} \min _{\Delta \mathbf{s} \neq \mathbf{0}} W_{r_{l}}(\Delta \mathbf{s}) .
\end{aligned}
$$

We remark that the above Min-Max criterion is also independently studied in [12] for VGR without considering the direct link. In the following, we shall obtain the diversity performances of both VGR and FGR by using a much simpler approach.

Proposition 1: The diversity order of both VGR and FGR using the Min-Max relay selection strategy (15) is $L+1$.

Proof: Let $W_{r_{l}}^{*}=\min _{\Delta \mathbf{s} \neq \mathbf{0}} W_{r_{l}}(\Delta \mathbf{s})$ and $W_{r_{q}}^{*}=$ $\max _{l=1,2, \cdots, L} W_{r_{l}}^{*}$, then we have

$$
F_{W_{r_{q}}(\Delta \mathbf{s})}(w) \leq \operatorname{Pr}\left(W_{r_{q}}^{*} \leq w\right) \leq \prod_{l=1}^{L} \sum_{\Delta \mathbf{s} \neq \mathbf{0}} F_{W_{r_{l}}(\Delta \mathbf{s})}(
$$

For VGR, we have $W_{r_{l}, F G R}(\Delta \mathbf{s})$

$\frac{P^{2} \lambda_{s r} \lambda_{r d}\left|g_{l}\right|^{2}\left|\mathbf{f}_{s l}^{T} \Delta \mathbf{s}\right|^{2}}{4\left(P \lambda_{r d}\left|g_{l}\right|^{2}+K P G \lambda_{s r}+1\right)} \stackrel{P \rightarrow \infty}{\geq} \frac{P \lambda_{s r} \lambda_{r d}\left|g_{l}\right|^{2}\left|\mathbf{f}_{s l}^{T} \Delta \mathbf{s}\right|^{2}}{4\left(\lambda_{r d}\left|g_{l}\right|^{2}+K G \lambda_{s r}+1\right)}$

$\mathrm{CDF}$ of $W_{r_{l}, F G R}(\Delta \mathbf{s})$ is thus given by

$$
F_{W_{r_{l}, F G R}(\Delta \mathbf{s})}(w) \stackrel{P \rightarrow \infty}{\leq} \frac{c_{3}}{a_{3} b_{3}} \frac{w}{P} \log \left(\frac{P}{w}\right),
$$

where $a_{3}=\lambda_{r d}, \quad b_{3}=\frac{\lambda_{s r}\|\Delta \mathbf{s}\|^{2}}{4}, \quad c_{3}=$ $K G \lambda_{s r}+1$ and the approximation is due to (9). Likewise, for VGR we have $W_{r_{l}, V G R}(\Delta \mathbf{s})=$ $\frac{P^{2} \lambda_{s r} \lambda_{r d}\left|g_{l}\right|^{2}\left|\mathbf{f}_{l}^{T} \Delta \mathbf{s}\right|^{2}}{4\left(P \lambda_{r d}\left|g_{l}\right|^{2}+P \lambda_{s r} \sum_{k=1}^{K}\left|f_{k l}\right|^{2}+1\right)} \stackrel{P \rightarrow \infty}{\geq} \frac{P \lambda_{s r} \lambda_{r d}\left|g_{l}\right|^{2}\left|\mathbf{f}_{s l}^{T} \Delta \mathbf{s}\right|^{2}}{4\left(\lambda_{r d}\left|g_{l}\right|^{2}+\lambda_{s r} \sum_{k=1}^{K}\left|f_{k l}\right|^{2}+1\right)}$
The CDF of $W_{r_{l}, V G R}(\Delta \mathbf{s})$ is given by

$$
F_{W_{r_{l}, V G R}(\Delta \mathbf{s})}(w) \stackrel{P \rightarrow \infty}{\leq} \frac{(K-1) c_{4}+1}{a_{4} b_{4}} \frac{w}{P} \log \left(\frac{P}{w}\right)
$$

where $a_{4}=\lambda_{r d}, b_{4}=\frac{\lambda_{s r}\|\Delta s\|^{2}}{4}, c_{4}=\lambda_{s r}$, and we use the small value approximation in (8). Plugging (17) and (18) back into (16) leads to $F_{W_{r_{q}}(\Delta \mathbf{s})}(w) \stackrel{P \rightarrow \infty}{\leq} C\left[\frac{w}{P} \log \left(\frac{P}{w}\right)\right]^{L}$, where $C$ is some constant. Therefore, for both of VGR and FGR we can obtain

$$
\begin{aligned}
& E\left[\exp \left(-W_{r_{q}}(\Delta \mathbf{s})\right)\right] \\
& \stackrel{P \rightarrow \infty}{\leq} C P(-1)^{L} \int_{0}^{\infty} \exp (-P w) w^{L}(\log w)^{L} d w \\
& \left.\stackrel{(a)}{=} C P(-1)^{L} \frac{\partial^{L}}{\partial w^{L}}\left\{P^{-w} \Gamma(w)\right\}\right|_{w=L+1} \\
& \left.\stackrel{P \rightarrow^{\infty}}{\approx} C P(-1)^{L}\left\{\frac{\partial^{L} P^{-w}}{\partial w^{L}} \Gamma(w)\right\}\right|_{w=L+1} \\
& =C \Gamma(L+1) \frac{(\log P)^{L}}{P^{L}},
\end{aligned}
$$

where we use $[14,4.358 .5]$ in (a), and $\Gamma(x)$ is the Gamma function $[14,8.310 .1]$. Combining the above result with (7) completes the proof.

\section{SIMULATIONS}

In this section, we shall present some simulation results to validate our analysis. Throughout simulations, we use the path loss model $\lambda=D^{-3}$, where $\lambda$ is the channel gain and $D$ is the distance between two terminals. Pair error probability is used as the performance metric, i.e., the probability that at least one of the source symbols is decoded incorrectly at the destination. To simplify the simulation settings, only symmetric networks with one or two users are considered, and $D_{s d}$ is always normalized to 1 .

Fig. 1 shows the error performances with different channel conditions, where there are two users using QPSK signal and there is only one relay node. Compared with direct transmission (DT), a diversity order of 2 is achieved due to node cooperation. We observe that VGR has about $1 \mathrm{~dB}$ SNR gain over FGR in all cases. It is also observed that the error performances almost keep unchanged after improving the qualities of user-relay channels, whereas about 3dB SNR gain is achieved when the relay-destination channels become better, which is consistent with our analysis that the relay-destination channel conditions dominate the error performances.

We also compare the single-user network with two-user network using the same total data rate in Fig. 2 after properly normalizing the transmitted power, where $D_{s r}=D_{r d}=0.5$. We observe that full diversity is achieved in all cases. However, single-user VGR is superior to all the other schemes when the total data rate is only 1 bit per channel use (bpcu) because there is no diversity loss in the reasonable SNR regions. When the data rate is $2 \mathrm{bpcu}$, although the error probability of single-user VGR still decreases faster, the spectral efficiency dominates 


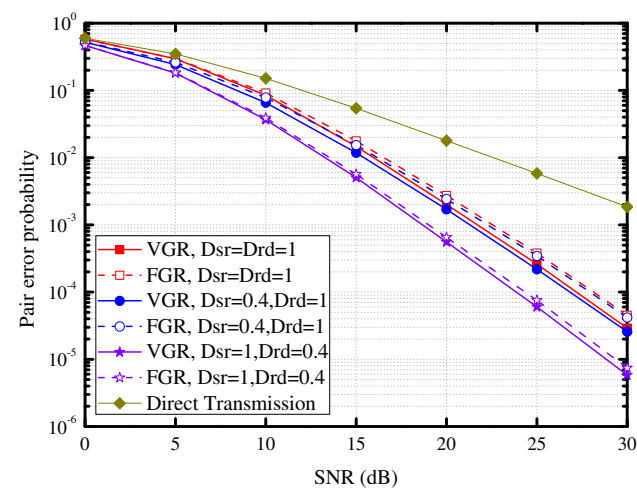

Fig. 1. Error performances of a two-user network with different channel conditions.

the performances at reasonable SNRs and thus the two-user system shows huge performance gain.

Next we study the error performances of relay selection in Fig. 3, where QPSK signal is employed. As the reference, we also simulate the Ding's scheme [9] for VGR, where $q=\arg \max _{l=1,2, \cdots, L} \frac{P \lambda_{r d}\left|g_{l}\right|^{2}\left(P \lambda_{s r} \sum_{k=1}^{K}\left|f_{k l}\right|^{2}+1\right)}{P \lambda_{r d}\left|g_{l}\right|^{2}+P \lambda_{s r} \sum_{k=1}^{K}\left|f_{k l}\right|^{2}+1}$. Clearly, our Min-Max scheme can achieve full diversity for both of VGR and FGR, whereas the diversity of Ding's scheme is bounded by 2 . This is because in Ding's scheme, the channel phases have nothing to do with relay selection. However, the channel phases actually have tremendous effects on PEPs, since the source messages are randomly mixed in the air and the MUI depends largely on the orthogonality of the instant channel coefficients. Although Ding's scheme does achieve full diversity in terms of outage capacity [9], where successive interference cancelation is employed at the destination and error-free decoding is assumed, our Min-Max strategy is more practical for real systems where coding/decoding is no longer ideal.

\section{CONCLUSIONS AND FUTURE WORK}

In this work, we have analyzed the error performances of multiple access system using ANC. We revealed that the error performance is actually dominated by the relay-destination channel, and the best relay position should be close to the destination. We also proposed a relay selection strategy based on Max-Min criterion and proved that full diversity can be achieved. In future work, we may further study the multiple access system using PLNC instead.

\section{REFERENCES}

[1] K. J. R. Liu, A. K. Sadek, W. Su, and A. Kwasinski, Cooperative Communications and Networking, Cambridge University Press, 2008.

[2] J. N. Laneman, D. N. C. Tse, and G. W. Wornell, "Cooperative Diversity in Wireless Networks: Efficient Protocols and Outage Behavior," IEEE Trans. Inf. Theory, vol. 50, no. 12, pp. 3062-3080, Dec. 2004.

[3] W. Su, A. K. Sadek, and K. J. R. Liu, "Cooperative Communications in Wireless Networks: Performance Analysis and Optimum Power Allocation," Wireless Personal Commun., vol. 44, no. 2, pp. 181-217, Jan. 2008.

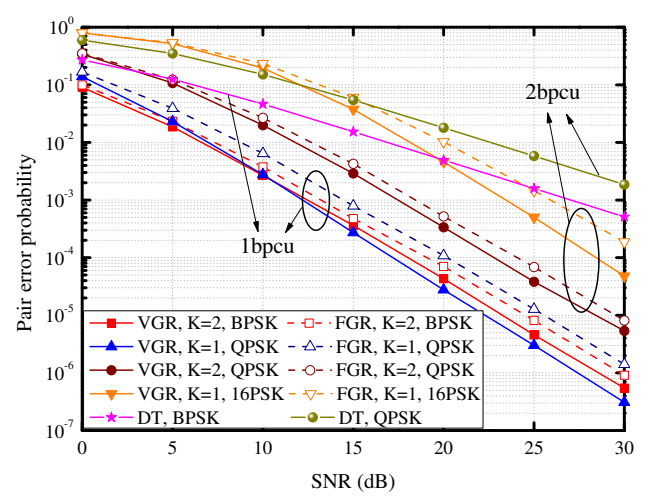

Fig. 2. Comparison of two-user and single-user network with different data rate.

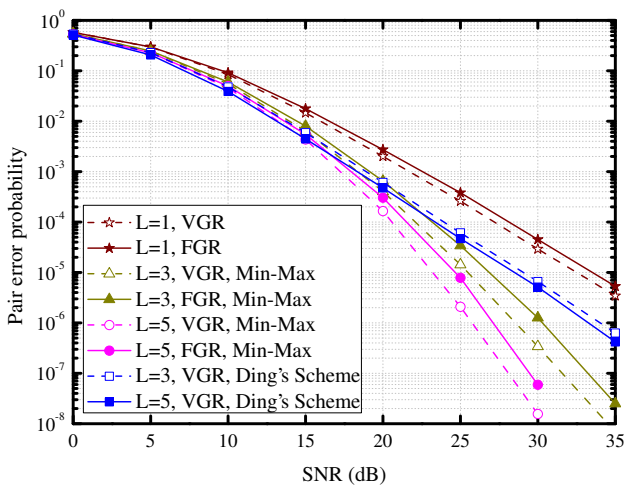

Fig. 3. Error performances of a two-user network with relay selection.

[4] M. O. Hasna and M.-S. Alouini, "A Performance study of dual-hop transmissions with fixed gain relays," IEEE Trans. Wireless Commun., vol. 3, no. 6, pp. 1963-1968, Nov. 2004.

[5] S. Katti, S. Gollakota, and D. Katabi, "Embracing wireless interference: Analog network coding," in ACM SIGCOMM 2007, Aug. 2007.

[6] S. Zhang, S. C. Liew, and P. P. Lam, "Physical-Layer Network Coding," in ACM MOBICOM 2006, Los Angeles, Sept. 2006.

[7] W. Guan and K. J. R. Liu, "Performance Analysis of Two-Way Relaying with Non-Coherent Differential Modulation," IEEE Trans. Wireless Commun., vol. 10, no. 6, pp. 2004-2014, June 2011.

[8] W. Guan and K. J. R. Liu, "On The Design of Relaying and Detection Schemes to Mitigate Error Propagation with Wireless Network Coding," submitted to IEEE Trans. Wireless Commun., Nov. 2011.

[9] Z. G. Ding, T. Ratnarajah, and Kin K. Leung, "On the Study of Network Coded AF Transmission Protocol for Wireless Multiple Access Channels," IEEE Trans. Wireless Commun., vol. 8, no. 1, pp. 118-123, Jan. 2009.

[10] D. Q. Chen, K. Azarian, and J. N. Laneman, "A Case for AmplifyForward Relaying in the Block-Fading Multiple-Access Channel," IEEE Trans. Inf. Theory, vol. 54, no. 8, pp. 3728-3733, Aug. 2008.

[11] M. Badr and J. C. Belfiore, "Distributed Space Time Codes for the Amplify-and-Forward Multiple-Access Relay Channel," in ISIT 2008, pp. 2543-2547, July 2008.

[12] E. Koyuncu and H. Jafarkhani, "Distributed Beamforming in Wireless Multiuser Relay-Interference Networks with Quantized Feedback," [Online] Available: http://arxiv.org/abs/1007.5514.

[13] M. Abramovitz and I. A. Stegun, Handbook of Mathematical Functions with Formulas, Graphs, and Mathematical Tables, 9th ed. New York: Dover, 1972.

[14] I. S. Gradshteyn and I. M. Ryzhik, Table of Integrals, Series, and Products, 7th ed. New York: Academic, 2007.

[15] W. Guan and K. J. R. Liu, "On Diversity Analysis of Analog Network Coding with Multi-User Interferences," submitted to IEEE Trans. Wireless Commun., Dec. 2011. 